\title{
Rhabdomyolysis after intramuscular iron-dextran in malabsorption
}

\author{
W D Foulkes, C Sewry, J Calam, H J F Hodgson
}

\begin{abstract}
The case of a 59 year old white man who had chronic malabsorption and selective IgA deficiency with severe iron deficiency is reported. In addition, he was deficient in vitamin $\mathbf{E}$ and selenium, important antioxidants which protect against lipid peroxidation. He was intolerant of oral iron and when treated with iron-dextran developed symptoms suggestive of polymyositis with evidence of rhabdomyolysis. It is suggested that free iron within iron-dextran activated free radicals, initiating lipid peroxidation and leading to polymyositis, rhabdomyolysis, and myoglobulinuria.
\end{abstract}

Iron-dextran has been used for many years to treat iron deficiency, either by intramuscular injection or by the total dose infusion technique. The iron-dextran (Imferon) contains $\mathbf{5 0}$ $\mathrm{mg} / \mathrm{ml}$ of iron with $20 \%$ dextran. It is isotonic and has a low ionic iron content. ${ }^{1}$ Local and systemic reactions are common-delayed arthralgia and myalgia are widely reported. ${ }^{2}$ Polymyositis or rhabdomyolysis have not previously been reported, however (Fisons Pharmaceuticals, Committee of Safety of Medicine, personal communication). We report here a patient with a severe and prolonged malabsorptive state who developed rhabdomyolysis during treatment with iron-dextran and suggest that he was predisposed to this owing to multiple deficiencies associated with his malabsorption.

\section{Case history}

The patient first presented to another hospital in 1982 with diarrhoea. He was noted to be iron deficient: haemoglobin $124 \mathrm{~g} / \mathrm{l}$, serum iron 3.0 $\mu \mathrm{mol} / \mathrm{l}$ (normal range $13-32$ ) with raised trans-

Department of Medicine, Royal Postgraduate Medical School Hammersmith Hospital, Du Cane Road, London W12 ONN W D Foulkes J Calam

J Calam

Jerry Lewis Muscle Research Centre, Royal Postgraduate Medical School, Hammersmith Hospital, London C Sewry

Correspondence to: Dr Hodgson. ferrin. His serum IgA was $0.42 \mathrm{~g} / \mathrm{l}$. Upper gastrointestinal endoscopy and radiology were normal, but a jejunal biopsy showed partial villous atrophy. There was no response to a gluten-free diet. His diarrhoea continued over the next few years and he did not receive any special diets or supplements. He was referred to the Hammersmith Hospital in 1987, where the above findings were confirmed. His HLA haplotype was A1 A2 B44 B15? DR3 DQ2 DRw52; smooth muscle antibodies positive 1 in 320 , antireticulin antibody negative. He made a good clinical response to oral prednisolone 20 $\mathrm{mg} /$ day with lessening of his diarrhoea. The jejunal biopsy did not show any significant improvement. Over the next year the dose was gradually reduced and his diarrhoea returned. He remained iron deficient. He stopped steroids altogether in February 1989.

He was unable to tolerate oral iron and therefore treatment was started in May 1989 with $2 \mathrm{ml}$ intramuscular iron-dextran. He had no ill effects. He received a further $5 \mathrm{ml}$ three days later and three further injections of $5 \mathrm{ml}$ were given weekly. He had marked myalgia at the sites of injection (alternate buttocks). Eighteen days after completing the course he noticed severe, generalised myalgia, which worsened over the next five days so that he was unable to lift his arms above his head or walk upstairs unaided. At this time he noted dark urine.

He was admitted to hospital. On examination he had profound proximal muscle weakness with myalgia. The rest of the examination was normal. Investigations on admission were as follows: haemoglobin $139 \mathrm{~g} / 1$, white cell count $6.3 \times 10^{9} / 1$, platelets $362 \times 10^{9} / 1$, creatinine 58 $\mu \mathrm{mol} / \mathrm{l}$. Creatine kinase $20400 \mathrm{IU} / \mathrm{l}$, aspartate transaminase $478 \mathrm{IU} / \mathrm{l}, \mathrm{C}$ reactive protein $28 \mathrm{~g} / \mathrm{l}$, erythrocyte sedimentation rate $33 \mathrm{~mm} / \mathrm{h}$. Prothrombin time 24 seconds, antiskeletal muscle antibody negative, anti-Ro, anti-La, and anti-Jol negative..Serum IgA $<0 \cdot 2 \mathrm{~g} / \mathrm{l}$, salivary IgA $<0.01 \mathrm{~g} / 1$. Vitamin E $6.6 \mu \mathrm{mol} / 1$ (normal range $11 \cdot 5-35$ ), selenium $0.81 \mathrm{nmol} / 1$ (normal range $1 \cdot 1-1 \cdot 9)$. Three day faecal fat $77 \cdot 3 \mathrm{~g} /$ day, urinary myoglobin strongly positive. A jejunal biopsy confirmed subtotal villous atrophy. The figure shows a deltoid muscle biopsy specimen obtained 19 days after the height of his myalgia. The patient made an uneventful recovery from the polymyositis and did not receive steroids. Twenty four days after the onset of the generalised myalgia his creatine kinase was $134 \mathrm{IU} / \mathrm{l}$.

\section{Pathological findings}

MUSCLE BIOPSY

A muscle biopsy specimen was taken from the right deltoid muscle under local anaesthesia with conchotome forceps. After transverse orientation the sample was rapidly frozen in isopentane cooled in liquid nitrogen. Cryostat sections $(6 \mu \mathrm{m})$ were stained histologically and histochemically with a variety of techniques to show the general morphology and fibre typing. ${ }^{3}$ In addition, serial sections were labelled immunocytochemically with antibodies to HLA $\mathrm{ABC}$ class $\mathrm{I}$ antigens and myosin isoforms, using a biotinylated secondary antibody followed by streptavidin conjugated to the fluorochrome Texas red.

The muscle biopsy specimen showed varia- 

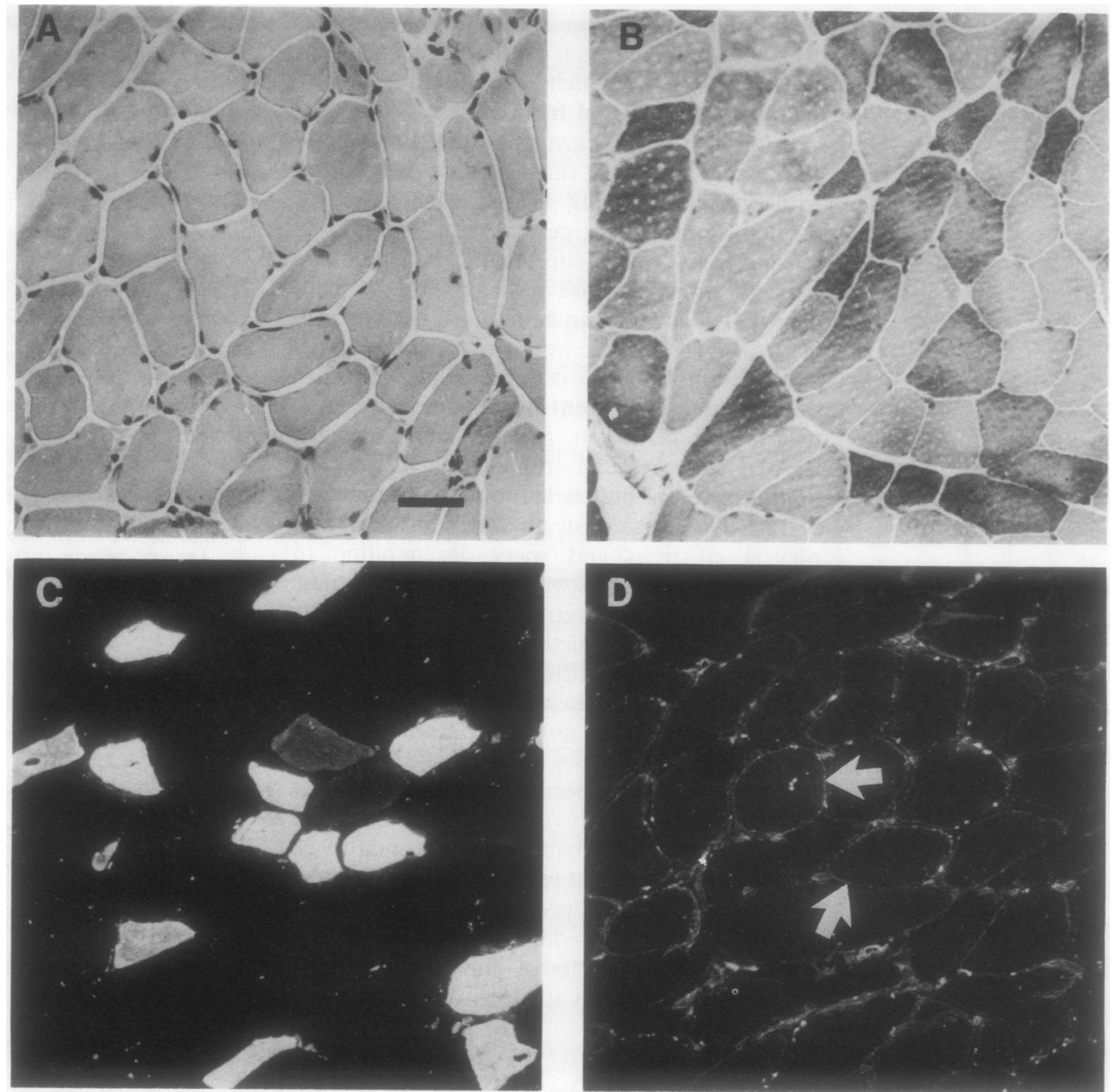

(A) Section stained with haematoxylin and eosin showing variation in fibre size but no necrosis, increase in connective tissue or cellular infiltrate. (B)Myosin ATPase ( $\mathrm{pH}$ 9.4) showing more marked variation in the darkly stained type 2 fibres. $(C)$ Section immunolabelled with antibodies to fetal myosin showing a population of small positive regenerating fibres. The bright punctate areas at the periphery of many fibres are due to autofluorescence of lipofuscin. (D) Immunolabelling of HLA class I expression. This is present on the periphery of several fibres (arrows).

tion in fibre size (figure A), particularly of the type 2 fibres (figure B). Type 1 fibres were predominant, and oxidative enzyme staining showed a population of small lobulated fibres with prominent peripheral mitochondrial aggregates. Basophilic fibres were apparent, and several small fibres were positively labelled with antibodies to a fetal isoform of myosin (figure C). Immunostaining for HLA class I antigens showed that the periphery of several fibres was positive (figure $\mathrm{D}$ ). There was no necrosis and no increase in cellularity or connective tissue.

The presence of a population of immature fibres suggests that the muscle was regenerating after an episode of muscle damage. Normal muscle fibres do not express detectable levels of HLA class I antigens, but they commonly occur on the surface of muscle fibres in inflammatory myopathies. ${ }^{4}$ The presence of HLA class I antigens on several fibres in this biopsy specimen supports the clinical evidence of myositis before regeneration.

\section{Discussion}

The clinical and laboratory evidence indicates that this patient experienced rhabdomyolysis.
Although we cannot exclude the possibility that this was a brief episode of autoimmune polymyositis, the short clinical course and the absence of lymphocytic infiltrate in the biopsy specimen argue against this. He had no antecedent viral illness, did not undertake any excessive physical effort, and did not consume large amounts of alcohol immediately before his generalised myalgia.

We propose that intramuscular iron played a critical part in the development of muscle damage in this man. Owing to prolonged severe malabsorption his iron deficiency was associated with profound deficiencies of vitamin $E$ and selenium, each of which protect against oxidative damage. Under these circumstances the free iron in iron-dextran might have initiated oxidative injury to muscle membranes. A similar argument has been invoked to explain the observation that intravenous iron can exacerbate rheumatoid arthritis; Blake and colleagues arguing that the synovitis that occurs is mediated by the free iron present in iron-dextran. ${ }^{5}$

Iron generates potent hydroxyl radicals through the Fenton reaction:

$$
\mathrm{H}_{2} \mathrm{O}_{2}+\mathrm{Fe}^{2+} \rightarrow \mathrm{OH}^{\bullet}+\mathrm{OH}^{-}+\mathrm{Fe}^{3+}
$$


Iron-dextran can saturate phosphate esters, organic acids, and iron storage/transport proteins, many of which can catalyse DNA degradation by way of free radicals. ${ }^{6}$

Vitamin $\mathrm{E}$ is the most important lipid phase antioxidant and may function in collaboration with selenium, acting in the aqueous phase through glutathione peroxidase. ${ }^{7}$ Vitamin E scavenges hydroxyl, superoxide, and peroxide radicals, which oxidise $\alpha$ tocopherol to tocopheryl. Tocopheryl can be reduced back by way of ascorbic acid, which would in turn be oxidised.

Furthermore, there is experimental evidence that vitamin E deficient piglets are susceptible to iron administration. Studies from Denmark showed that $2 \mathrm{ml}$ of iron complex $\left(\mathrm{Fe}^{3+} 75\right.$ $\mathrm{mg} / \mathrm{ml}$ ) given to piglets led to death in nine out of $17 .^{8}$ The cause of death was pericardial and pleural effusions and waxy degeneration of skeletal muscle. The sow's milk was found to be low in vitamin E. Pretreatment of the piglets with vitamin E completely abolished this toxic effect in subsequent litters.

What explanation do we have for the fact that this phenomenon has not been described before despite widespread use of iron-dextran? This might be due to underreporting as myalgia is common but rarely investigated and therefore minor degrees of rhabdomyolysis might go unnoticed. The patient reported here is unusual, however, as he had prolonged malabsorption leading to numerous deficiencies over several years and only partially and intermittently responded to medical treatment, so that other deficiencies were present; this would not be true of most patients receiving iron-dextran injections. Furthermore, selective IgA deficiency may permit absorption of luminal antigens, ${ }^{9}$ some of which might also contribute towards the susceptibility of muscle to free radical damage.

We thank Fisons Pharmaceuticals for discussing this case, Dr David Muller for performing the vitamin $\mathrm{E}$ assay and for helpful suggestions, Dr Wendy Brown, who provided the antibodies to fetal myosin, the Muscular Dystrophy Group of Great Britain and Northern Ireland, who supported our work, and Miss F Nadiadi for preparing the manuscript.

1 Cox J S G, King R E, Reynolds G F. Valency investigation

2 Wallerstein R O. Intravenous iron-dextran complex. Blood 1968; 32: 690-5.

3 Dubowitz V. Muscle biopsy: a practical approach. 2nd ed. London: Baillière Tindall, 1985.

4 McDougall T M, Dunn M J, Dubowitz V. Expression of class I and class II MCH antigens in neuromuscular disease. f Neurol Sci 1989; 89: 213-26.

5 Blake D R, Lunec J, Ahem M, Ring E F J, Bradfield J, Gutteridge J M C. The effect of intravenous iron-dextran 6 on rheumatoid arthritis. Ann Rheum Dis 1985; 44: 183-8. Oxygen free radicals, inflammation and synovitis: the Oxygen free radicals, inflammation and synovits
current status. Ann Rheum Dis 1989; 48: 864-70.

7 Rotruck J T, Pope A L, Swanson A B, Hafeman D G Hoekstra W G. Selenium: biochemical role as a component of glutathione peroxidase. Science 1973; 179: 588-90.

8 Lannek $\mathrm{N}$, Lindberg $\mathrm{P}$, Tollerz $\mathrm{G}$. Lowered resistance to iron in vitamin E deficient piglets and mice. Nature 1962; 195: 1006-7.

9 Fubara E S, Freter R. Protection against enteric bacterial infection by secreting IgA antibodies. F Immunol 1973; 111 395-403.

10 Brandtzaeg P, Valnes K, Scott H, Rognum T O, Bjerke K, Baklein $\mathrm{K}$. The human gastrointestinal secretory immune system in health and disease. Scand $\mathcal{F}$ Gastroenterol 1985 20: 17-18. 